\title{
Brief review of metamaterials and auxetic materials
}

\author{
Raluca NEGREA \\ University of Pitesti, Romania \\ *Corresponding author e-mail: raluca.negrea@yahoo.ro \\ Article history \\ Received 20.11.2020 \\ Accepted 05.01.2021 \\ DOI https://doi.org/10.26825/bup.ar.2021.003
}

\begin{abstract}
This paper aims to provide a comprehensive review on auxetic materials and structures, including various types of cellular auxetics, natural and artificial auxetics, metallic auxetics, multi-material and composite auxetics.

A material engineered to have a property that is not found in naturally occurring materials is the metamaterial (meaning"beyond" from the Greek word $\mu \varepsilon \tau \dot{\alpha}$ meta, and "material" from the Latin word materia) made from assemblies of multiple elements shaped from composite materials such as metals and plastics. They have exceptional properties, derive from their geometrical shape rather than directly from the behavior of the base materials, in terms of mechanical response, energy absorption and, heat transport performance.

One of the most studied species of mechanical metamaterials is the auxetic materials, a type of mechanical metamaterial with a negative Poisson's ratio (which shrink transversely under longitudinal compression and expand transversely under longitudinal tension). They consist of numerous hinge-like cells that are joined together. The cells have a re-entrant geometry, i.e. under pressure, they expand in lateral direction. Superior and unusual properties of auxetics are presented and some existing or potential applications are summarized.

Accompanied by uncommon deformation pattern under compression and tension, auxetic materials and structures are endowed with many desirable material properties, such as superior shear resistance, indentation resistance, fracture resistance, synclastic behavior, variable permeability and better energy absorption performance.
\end{abstract}

Keywords: Metamaterials; Auxetic; Young's modulus; Poisson coeficient

\section{Introduction}

Metamaterials is a new field of interdisciplinary research, which deals with artificial material composites engineered to display physical properties that surpass (or complement) those available in nature. Originally proposed as a way of tailoring media's electromagnetic and optical properties, the metamaterial concept has recently extended its reach to also include elastic, acoustic, and thermal properties.[1][2] Since 1999, with the emergence of the field, metamaterials has attracted a lot of attention from the scientific community all over the globe, offering immediate applications in antenna and waveguide engineering, imaging, microscopy, sensing, and light manipulation.[3]

A metamaterial (from the Greek word $\mu \varepsilon \tau$ ó meta, meaning "beyond" and the Latin word materia, meaning "matter" or "material") is a material engineered to have a property that is not found in naturally occurring materials.[4][5] They are made from assemblies of multiple elements fashioned from 
composite materials such as metals and plastics. The materials are usually arranged in repeating patterns, at scales that are smaller than the wavelengths of the phenomena they influence. Metamaterials derive their properties not from the properties of the base materials, but from their newly designed structures. [6] Their precise shape, geometry, size, orientation and arrangement gives them their smart properties capable of manipulating electromagnetic waves: by blocking, absorbing, enhancing, or bending waves, to achieve benefits that go beyond what is possible with conventional materials. [7][8]

Recently, artificially constructed metamaterials have become of considerable interest, because these materials can exhibit electromagnetic characteristics unlike those of any conventional materials. Artificial magnetism and negative refractive index are two specific types of behavior that have been demonstrated over the past few years, illustrating the new physics and new applications possible when we expand our view as to what constitutes a material. [9][10]

Typical mechanical metamaterials are materials with negative indexes, e.g., NPR, negative compressibility (NC), or negative normal stress. As a most studied branch of mechanical metamaterials, auxetic materials exhibit counterintuitive deformation behaviour during deformation. To be more specific, under uniaxial compression (tension), conventional materials expand (contract) in the directions orthogonal to the applied load. In contrast, auxetic materials contract (expand) in the transverse direction, as shown in figure 1. Numerous desirable properties resulting from this unusual behaviour have been attracting an increasing numberof researchers to the field of auxetics materials and structures. [11]

\section{Tension}

(a)
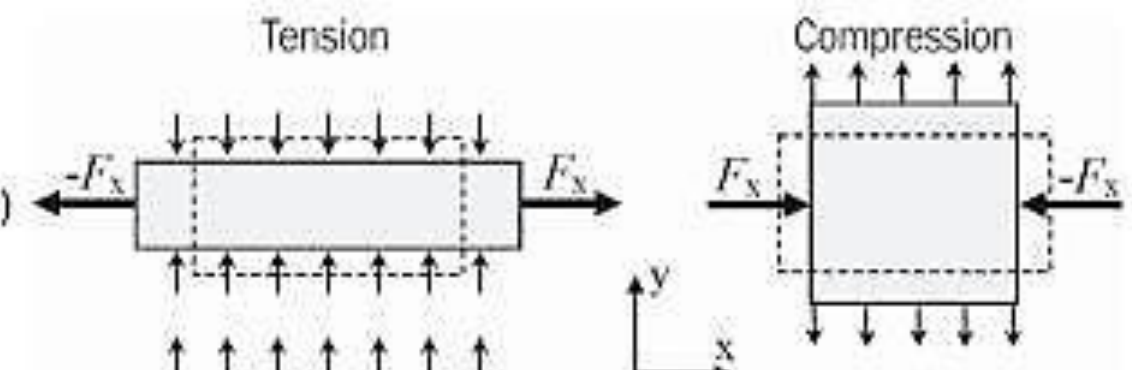

(b)
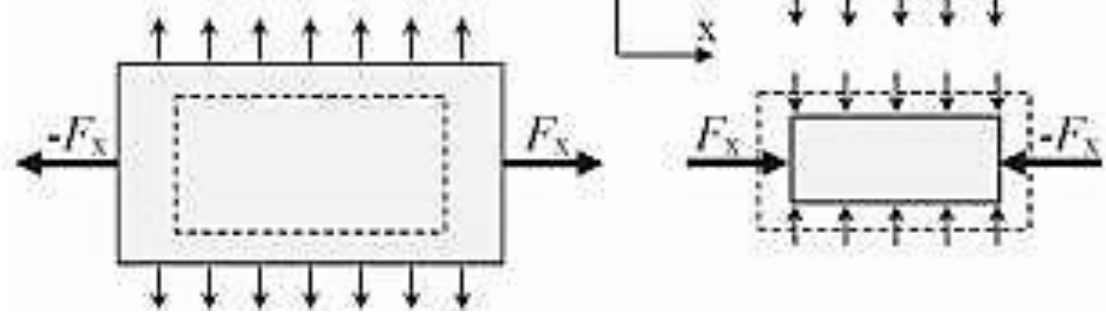

Figure 1. Behaviors in tensile and compressive load (a) non-auxetic material; (b) auxetic material

Accompanied by uncommon deformation pattern under compression and tension, auxetic materials and structures are endowed with many desirable material properties, such as superior shear resistance, indentation resistance, fracture resistance, synclastic behavior, variable permeability and better energy absorption performance. These materials have a negative Poisson's ratio, $v$, which means that they expand in the lateral direction when stretched longitudinally i.e. in simple terms, they get fatter when pulled. This is a novel interesting effect in itself but it is the consequence of having a negative $v$ that marks these materials as being candidate aerospace solutions for the future. [12][13] From classical elasticity theory, many properties depend on the Poisson's ratio of the material. A simple and well known example of this is the shear modulus, $G$, where:

$$
\mathrm{G}=\frac{E}{2(1+v)}
$$

and $\mathrm{E}$ is the Young's modulus. It can clearly be seen that as $v$ approaches -1 , which is the limit for an isotropic material, the shear modulus will become infinitely large.[14][15]

The Poisson's ratio is defined as the negative ratio of lateral strain to the stretched longitudinal strain. Tensile deformation is determined as positive and compression deformation is determined as negative. As the formula of the Poisson's ratio contains a minus sign, most known materials have a positive ratio reflecting a tendency to conserve volume. According to the theory of elastic mechanics, $\mathrm{v}$ is between 0 and 0.5 . It is close to 0.3 for many materials and approximately 0.5 for rubbery materials. The material tries to maintain its density causing a contraction in the lateral direction. Materials with a high atomic 
packing density such as gold have a high Poisson's ratio of 0.5 , while materials which have a lower atomic packing density such a crystallite metals have a Poisson's ratio of around 0.3 .

Auxetic materials are still an emerging class of materials. They consist of numerous hinge-like cells that are joined together. The cells have a re-entrant geometry, i.e. under pressure, they expand in lateral direction. The word re-entrant refers to an angle in a polygon that is greater than $180^{\circ}$ and therefore pointing inwards. The side-way expansion is responsible for the negative Poisson's ratio of auxetic materials. A homogeneous, isotropic 3D solid has a potential Poisson's ratio between -1.0 and 0.5 . This range can be enlarged for anisotropic solids to more extreme values. [16][17]

Research into auxetics began in earnest in the late 1980s with the fabrication of auxetic polyurethane foam. Since then, a wide range of materials have been produced covering the major classes of materials (polymers, composites, metals, and ceramics). Practically, to be auxetic indicates that a structure/material expands when stretched and contracts laterally when compressed, in contraposition to a positive Poisson's ratio one, like rubber, who contracts when stretched and becomes in bulge when compressed.

Such materials are called also dilatational materials because they exhibit substantial volumetric changes when loaded. First auxetic cellular structures were created as 2D silicone rubber or aluminum honeycombs and were extensively investigated by Lakes in his many publications. Anti-rubber material was also found to be used to refer to this kind of structures and materials.[18][19][20]

The words "auxetic model" will be used to refer to analytical and mathematical geometries that propose spatial configurations of elements ideally behaving in an auxetic way. The models consist into the abstraction of realizable organisms. Being abstract they don't need to have a link to any specific material or to a production process, dimensions or physical properties. But nothing implies that they can't be use to theorize functioning technologies and complex organisms.[21]

\section{Discussion}

Due to the negative Poisson's ratio, auxetic materials have enhanced mechanical properties Table 1, most of which are superior to similar properties of non-auxetic materials, and some of them unique. Auxetic materials are suitable for many practical applications. The main existing and potential applications of auxetic materials are presented in Table 2.[17][21]

Figure 2 illustrates a general classification of auxetic materials. Auxetic materials exist in nature in two forms: biological and mineral. Depending on the deformation direction, synthetic or man-made auxetic materials under mechanical load may be subdivided into two main groups: two-dimensional and threedimensional. The most known natural auxetic materials are presented on figure 3 .

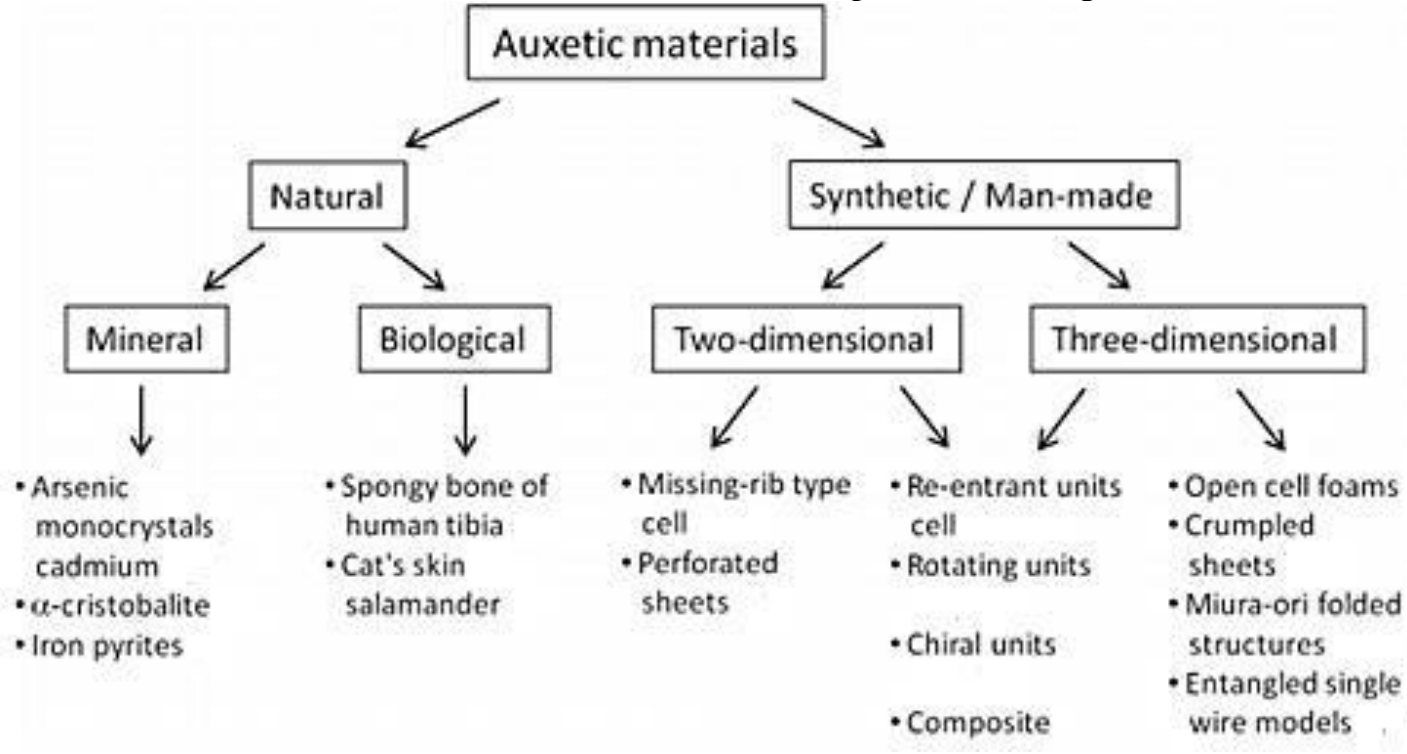

Figure 2. General classification of auxetic materials.[22] 
Table 1. Mechanical properties of auxetic materials.[22][20]

\begin{tabular}{|c|c|}
\hline $\begin{array}{c}\text { Mechanical } \\
\text { properties }\end{array}$ & Description \\
\hline $\begin{array}{l}\text { Increased } \\
\text { Energy } \\
\text { Absorption }\end{array}$ & $\begin{array}{l}\text { As it is shown by experimental results, auxetic materials exhibit a better damping } \\
\text { and sound absorption (ex: Porous auxetic foams) compared to conventional } \\
\text { materials }\end{array}$ \\
\hline $\begin{array}{l}\text { Enhanced } \\
\text { Indentation } \\
\text { Resistance }\end{array}$ & $\begin{array}{l}\text { This property is the result of two main reasons: high shear stiffness, and the fact that } \\
\text { when an impactor touches their surfaces, auxetic materials move to regions where } \\
\text { the impact is applied, what increases the density of these regions. Many studies have } \\
\text { been carricd out on materials with re-entrant unit cells, polymeric and metallic } \\
\text { foams, and composite sandwich panels }\end{array}$ \\
\hline $\begin{array}{l}\text { High Shear } \\
\text { Stiffness }\end{array}$ & $\begin{array}{l}\text { From the well know relation between the Poisson's ratio ( } v) \text {, the Young's modulus } \\
(E) \text {, shear modulus }(G) \text {, the following conclusion could be mad: at negative } \\
\text { magnitudes of the Poisson's ratio, the shear modulus becomes higher. When the } \\
\text { value of the Poisson's ratio approaches close to }-1 \text {, the shear modulus tends to } \\
\text { infinity, and the shear resistance gets significantly larger. Researches on this } \\
\text { property have been carricd out on structures with re-entrant and chiral unit cells }\end{array}$ \\
\hline $\begin{array}{l}\text { High } \\
\text { Fracture } \\
\text { Toughness }\end{array}$ & $\begin{array}{l}\text { Conducted studies have shown that it requires more energy to propagate a crack in } \\
\text { auxetic material, and that toughness could be changed as the Poisson's ratio varies, } \\
\text { and therefore the material becomes very tough if the Poisson's ratio gets close to }-1 \text {. } \\
\text { Many studies have been conducted on structures with re-entrant unit cells, } \\
\text { composite sandwich panels and solid foams }\end{array}$ \\
\hline $\begin{array}{l}\text { Synclastic } \\
\text { Curvature } \\
\text { in Bending }\end{array}$ & $\begin{array}{l}\text { Under bending load applied on two opposite sides, auxetic surfaces undergo } \\
\text { synclastic deformation. This property allows the manufacturing of structures with } \\
\text { different shapes through a relatively small number of machining steps, avoiding the } \\
\text { waste of material, and consequently with low manufacturing cost. This feature is } \\
\text { most typical for structures with re-entrant unit cells }\end{array}$ \\
\hline $\begin{array}{l}\text { Variable } \\
\text { Permeability }\end{array}$ & $\begin{array}{l}\text { Porous auxetic materials are suitable for varying permeability. This property allows } \\
\text { to control the permeability of filters. When a load is applied in a certain direction, } \\
\text { the size of their pores changes. In case of tensile load, their size increases. This } \\
\text { variable permeability can be used from macroseale to nanoseale materials. All } \\
\text { porous auxetic materials exhibit this property }\end{array}$ \\
\hline
\end{tabular}

Table 2. Aplications of auxetic materials.[22]

Field Application (existing and potential)

\begin{tabular}{ll}
\hline Aerospace & $\begin{array}{l}\text { Aircraft engine vanes, aireraft thermal protecting system, fasten belts, wing } \\
\text { (enhanced shear resistance) panel, aircraft nose-cones, rivet, sounds and } \\
\text { vibration absorber }\end{array}$ \\
Military (defense) & $\begin{array}{l}\text { Lighter protective materials, blast curtains, vehicle armor for ballistic } \\
\text { protection, helmet, bullet proof vest, protective gear, knec pad, protective gear } \\
\text { (better impact resistance) } \\
\text { Energy absorption devices, cushion, thermal protection, jounce bumper, } \\
\text { vehicle armor for ballistic protection, fastener } \\
\text { Composite reinforement (better adhesion between fiber and matrix) }\end{array}$ \\
Composite & $\begin{array}{l}\text { Stents, surgical implants, arterial prostheses, bandage, wound pressure pad, } \\
\text { auxetic scaffolds, health monitoring sensors, artificial skin, prosthetic linings, } \\
\text { sutures and ligament/muscle anchors } \\
\text { Reinforcement of masonry walls with an auxetic layer. The auxetic foams } \\
\text { used in this case, duc to their negative Poisson's ratios, increase the toughness } \\
\text { of the wall } \\
\text { Piezoclectric devices and composites, sensors/ hydrophone (higher sensitivity } \\
\text { and higher resistance variation in the sensor) } \\
\text { Comfortable textiles with reduced clothing pressure, textile fibers, functional } \\
\text { fabric, threads industry, color-change straps, or fabrics }\end{array}$ \\
\hline
\end{tabular}

As shown in Figure 3, the structural evolutions of various types of chiral mechanical metamaterials can be classified into chiral, anti-chiral and meta-chiral categories. Besides these periodic regular designs, mechanical properties of irregular and disordered chiral mechanical metamaterials are also investigated . Irregular chiral system possesses a lower level of rotational or axial symmetry, and exhibits highly 
anisotropic behavior, thus has the ability to exhibit a much wider range of mechanical properties in comparison to their regular counterparts.[23]

Among this general class of metamaterials, periodic chiral lattices have been shown to possess relatively compliant behavior because of their bending dominated response, while exhibiting considerable multiaxial expansion/contraction under uniaxial loads due to auxeticity. In addition, chiral honeycombs have been experimentally and numerically shown to possess Poisson's ratios in the range of $-1<v<0$. Among several two dimensional (2D) chiral lattices proposed in the literature, only the elastic properties of hexa- and tetra-chiral lattices have been investigated analytically, using micro-polar and secondgradient continuum theories.[24][25]

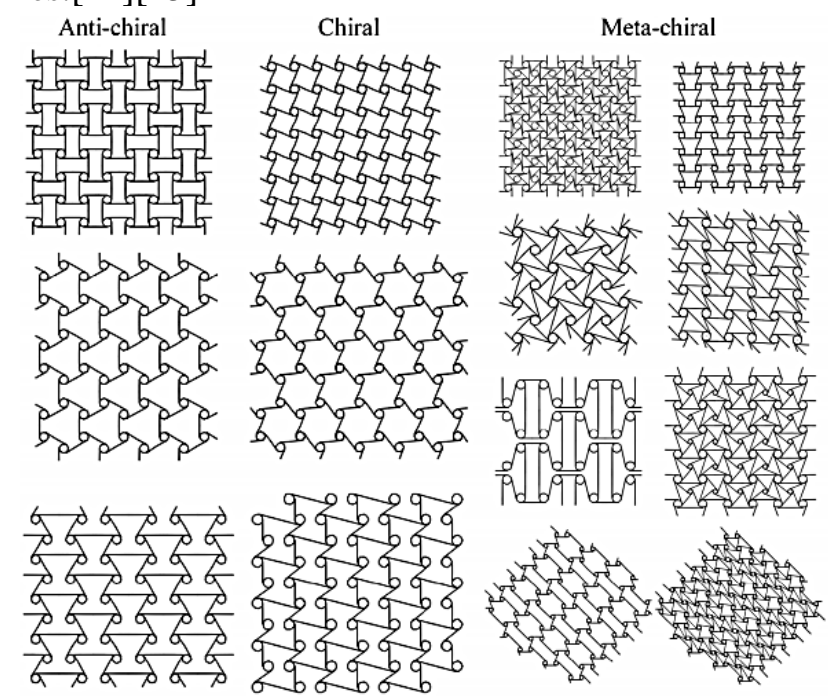

Figure 3. Classification of 2D chiral mechanical metamaterials.

These approaches are far more complex than the simple yet robust method used here for analytical study of chiral unit cells, which often require special boundary conditions at the unit cell level due to underlying rotational symmetry of the structure. [26]

According to the structural deformation models, these auxetic metastructures can be classified into following categories: missing-rib, rigid (or semi-rigid) rotation, re-entrant, chiral and elastic instability, etc. A more general classification of artificial auxetic mechanical metamaterials is shown in Figure 4.

(a)

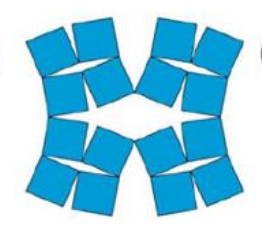

(e)

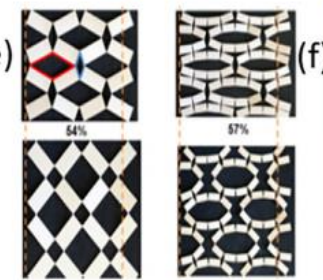

(b)
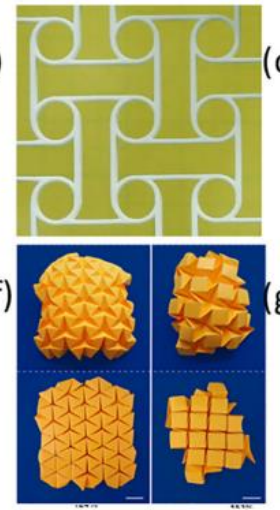
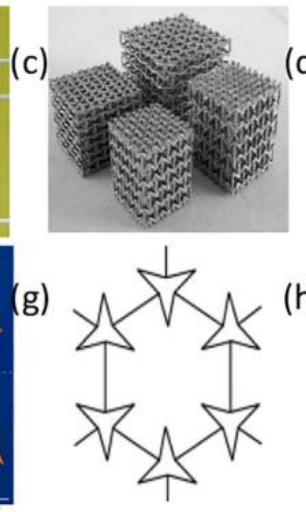

(d)

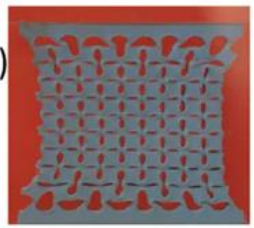

(h)

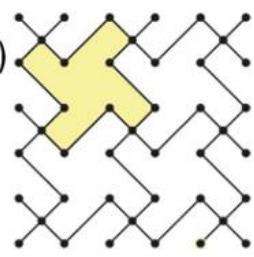

Figure 4. Classification of auxetics (a), rigid node rotation; (b) anti-tetrachiral; (c) re-entrant lattice; (d) elastic instability; (e) kirigami fractal cut; (f) origami; (g) star shape; (h) missing-rib model.

Owing to this unusual characteristic behavior, the materials with negative poisson's ratio have several advantages such as high shear modulus, synclastic curvature, high damping resistance, high fracture toughness, enhanced crack growth resistance and high energy absorption capability. [27]

It is possible to transform a non-auxetic material into auxetic materials by means of use different stacking sequences of individual lamina and also, lamina material should be highly anisotropic. Specially designed software are used to determine the stacking sequence and the software also can determine mechanical properties such as maximum stiffness, bending strength. Classification of auxetics by the production methods is presented on Figure 5.[28][20] 


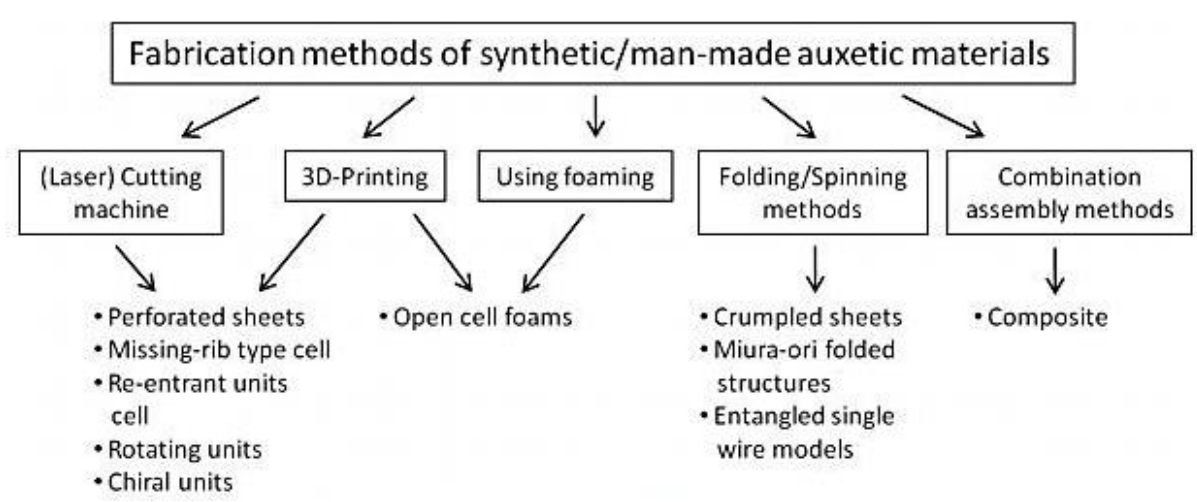

Figure 5. Fabrication methods of auxetic materials.

Auxetic composites have many advantages such as high specific stiffness, high specific strength and light weight. The auxetic composites have higher shear modulus, enhanced indentation resistance, synclastic curvature, better crack resistance, higher damping resistance than conventional composite materials. These advantages make auxetic composites very applicable for engineering applications, such as automotive and aerospace. Most parts of the aircraft are subjected to shear force, therefore, it is need to use the materials which have high shear modulus. [28][11]

.As shown in Figure 6, various types of chiral structures exist commonly in natural plants, animals and artificial synthesized materials, such as: helical goat horns, right-handed and left-handed sea shell, DNA, chiral carbon nanotube, twisting flower petals and stems, plant climbing tendrils and twisted leaves, chiral cellulose, etc. Chirality is encountered in many branches of science, including physics, biology, chemistry and optics. [16][29][9]It pervades much of modern science: from the physics of elementary particles, through organic stereochemistry, to the structure and behavior of the molecules of life, with much else besides (nonlinear optics, nanotechnology, materials, electrical engineering, pharmaceuticals, etc.). From mechanical and physical aspects of view, chiral material should be described by an adequate constitutive equation with handedness in order to characterize the distinct features.[24][7]

(a)
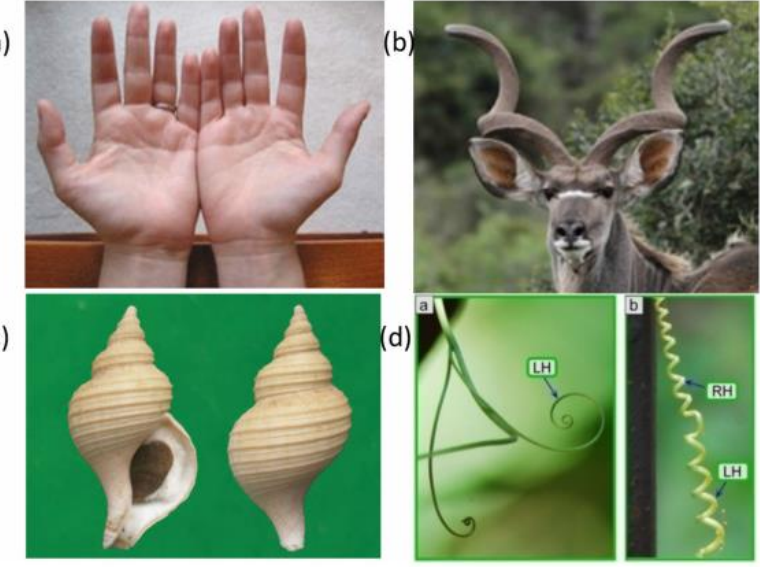

Figure 6. Chiral in nature: (a) right and left hands; (b) helical goat horns; (c) right-handed and lefthanded sea shell; (d) right-handed and left-hnded towel gourd tendrils

Remarkable progress has been made in the past three decades in the field of auxetics, including theoretical analysis, finite element simulations and experiments. However, many important and interesting problems still require further investigations. First of all, constrained by traditional manufacturing techniques, most of the previous studies of auxetics are based on simple 2D models. [30] Besides, although some 3D auxetic materials have been reported, most base materials of these 3D auxetics are rubber-like materials which could only sustain a very limited loading force and impact. [8][31] Apart from that, most of the existing 3D auxetic materials only exhibit NPR in a small effective strain range which greatly limits applications of these novel materials. More importantly, the geometries of the majority of the existing 3D auxetics are predesigned which often creates difficulty to tune their mechanical properties. Lastly, all reported auxetic tubular structures may not demonstrate auxetic behavior under compression which also constrains a wider application for auxetic tubular structures.[32][8][33][34] 
The evolution of important concepts related to chiral mechanical metamaterials is shown in Figure 7. Meanwhile, with the progress of advanced manufacturing techniques, various types of advanced additive manufacturing (AM) are widely employed for biomedical, aerospace, automotive, marine and offshore industrial sections. These low-cost AM techniques demonstrated superior manufacturing efficiencies and economic advantages for advanced lightweight industrial components with unlimited arbitrary topological layouts and complex internal microstructures, and are proposed for fabrication of auxetic materials and structures.[35][20]

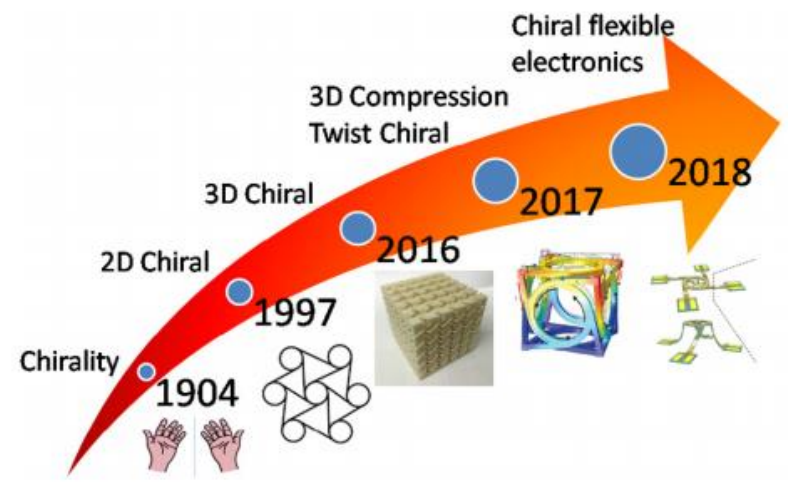

Figure 7. Important concepts on the development of chiral mechanical metamaterials

In order to fill the research gap of auxetics, a novel and recent methodology for generating 3D auxetic metallic materials is particularly reviewed, and successful cases are presented with numerical simulations and experiments.[15][27]

\section{Conclusion}

This paper aims to provide a comprehensive review on auxetic materials and structures, including various types of cellular auxetics, natural and artificial auxetics, metallic auxetics, multi-material and composite auxetics. Superior and unusual properties of auxetics are presented and some existing or potential applications are summarized.[22][24]

The cost of manufacturing auxetic materials is still too high. For most of the $3 \mathrm{D}$ auxetic materials with reliable auxetic behavior, the experimental specimens are fabricated using 3D printing technique. Although 3D printing technique is convenient in fabrication and enables engineers to concentrate on the design itself without considering too much of the manufacturing procedure, it is still a crucial problem to realise mass production with a lower cost which significantly constrains the wide application for auxetics.[29][1]

More importantly, all of the reviewed auxetic materials and auxetic structures have a substantial porosity in their geometrical configuration which inevitably reduces their mechanical capability when sustaining a load or impact. That is to say, obtaining some desirable properties of auxetic materials, such as enhanced shear resistance, improved indentation resistance and superior energy absorption, is actually at the cost of sacrificing the mechanical performance in the beginning when compared with solid materials. Therefore, in the most scenarios, the obtained auxetic behavior could not compensate for the loss of the mechanical performance resulting from the porous microstructure of auxetic materials which significantly decreases the advantages of the auxetic behavior, especially for the application of energy absorption or protective devices. However, the shortage of the porous microstructure of auxetic materials is rarely discussed.[36]

Although the properties of auxetics are very desirable, it is not very practical to bring them into the practical stage, especially when considering the cost performance. Also, these desirable properties of auxetic materials are actually not indispensable. This may explain that although a certain amount of auxetic materials have been designed and fabricated, very few of them have been used in the practical stage. Finally, several successful industrial application of chiral mechanical metamaterials are demonstrated, such as: morphing airfoil with chiral core configuration, shape memorial smart deployable antenna and programmable structures, auxetic stent for biomedical application, chiral flexible electronics and reconfigurable structures with shape shifting abilities, etc.[17][3] 
Although many engineering obstacles remain to be solved by materials with negative Poisson's ratio, this cannot happen before the suggested uses of these materials come to fruition commercially.

\section{References.}

[1] W. Wu, W. Hu, G. Qian, H. Liao, X. Xu, F. Berto, Mechanical design and multifunctional applications of chiral mechanical metamaterials: A review, Mater. Des. 180 (2019) 107950. https://doi.org/10.1016/j.matdes.2019.107950.

[2] X. Ren, R. Das, P. Tran, T.D. Ngo, Y.M. Xie, Auxetic metamaterials and structures: A review, Smart Mater. Struct. 27 (2018). https://doi.org/10.1088/1361-665X/aaa61c.

[3] J.U. Surjadi, L. Gao, H. Du, X. Li, X. Xiong, N.X. Fang, Y. Lu, Mechanical Metamaterials and Their Engineering Applications, Adv. Eng. Mater. 21 (2019) 1-37. https://doi.org/10.1002/adem.201800864.

[4] J.N. Grima, L. Mizzi, K.M. Azzopardi, R. Gatt, Auxetic Perforated Mechanical Metamaterials with Randomly Oriented Cuts, Adv. Mater. 28 (2016) 385-389. https://doi.org/10.1002/adma.201503653.

[5] H. Yang, L. Ma, Design and characterization of axisymmetric auxetic metamaterials, Compos. Struct. 249 (2020). https://doi.org/10.1016/j.compstruct.2020.112560.

[6] N.S. Ha, G. Lu, A review of recent research on bio-inspired structures and materials for energy absorption applications, Elsevier Ltd, 2020. https://doi.org/10.1016/j.compositesb.2019.107496.

[7] H. Ebrahimi, D. Mousanezhad, H. Nayeb-Hashemi, J. Norato, A. Vaziri, 3D cellular metamaterials with planar anti-chiral topology, Mater. Des. 145 (2018) 226-231. https://doi.org/10.1016/j.matdes.2018.02.052.

[8] Q. Wang, Z. Yang, Z. Lu, X. Li, Mechanical responses of 3D cross-chiral auxetic materials under uniaxial compression, Mater. Des. 186 (2020) 108226. https://doi.org/10.1016/j.matdes.2019.108226.

[9] D. Mousanezhad, B. Haghpanah, R. Ghosh, A.M. Hamouda, H. Nayeb-Hashemi, A. Vaziri, Elastic properties of chiral, anti-chiral, and hierarchical honeycombs: A simple energy-based approach, Theor. Appl. Mech. Lett. 6 (2016) 81-96. https://doi.org/10.1016/j.taml.2016.02.004.

[10] Y.Sun, Q.M. Li, Dynamic compressive behaviour of cellular materials: A review of phenomenon, mechanism and modelling, Int. J. Impact Eng. 112 (2018) 74-115. https://doi.org/10.1016/j.ijimpeng.2017.10.006.

[11] X. Ren, R. Das, P. Tran, T.D. Ngo, Y.M. Xie, Auxetic metamaterials and structures: A review, Smart Mater. Struct. 27 (2018). https://doi.org/10.1088/1361-665X/aaa61c.

[12] L.L. Hu, Z.R. Luo, Q.Y. Yin, Negative Poisson's ratio effect of re-entrant anti-trichiral honeycombs under large deformation, Thin-Walled Struct. 141 (2019) 283-292. https://doi.org/10.1016/j.tws.2019.04.032.

[13] J.E. Li, B.L. Wang, Effect of negative Poisson's ratio on the fracture mechanics parameters due to mechanical and thermal loads, Int. J. Eng. Sci. 150 (2020) 1-11. https://doi.org/10.1016/j.ijengsci.2020.103256.

[14] P.U. Kelkar, H.S. Kim, K.H. Cho, J.Y. Kwak, C.Y. Kang, H.C. Song, Cellular auxetic structures for mechanical metamaterials: A review, Sensors (Switzerland). 20 (2020). https://doi.org/10.3390/s20113132.

[15] X. Yu, J. Zhou, H. Liang, Z. Jiang, L. Wu, Mechanical metamaterials associated with stiffness, rigidity and compressibility: A brief review, Prog. Mater. Sci. 94 (2018) 114-173. https://doi.org/10.1016/j.pmatsci.2017.12.003.

[16] S. Linforth, T. Ngo, P. Tran, D. Ruan, R. Odish, Investigation of the auxetic oval structure for energy absorption through quasi-static and dynamic experiments, Int. J. Impact Eng. 147 (2021) 103741. https://doi.org/10.1016/j.ijimpeng.2020.103741.

[17] H.M.A. Kolken, A.A. Zadpoor, Auxetic mechanical metamaterials, RSC Adv. 7 (2017) 51115129. https://doi.org/10.1039/c6ra27333e.

[18] R. Hamzehei, S. Rezaei, J. Kadkhodapour, A.P. Anaraki, A. Mahmoudi, 2D triangular antitrichiral structures and auxetic stents with symmetric shrinkage behavior and high energy absorption, Mech. Mater. 142 (2020) 103291. https://doi.org/10.1016/j.mechmat.2019.103291. 
[19] C. Qi, F. Jiang, C. Yu, S. Yang, In-plane crushing response of tetra-chiral honeycombs, Int. J. Impact Eng. 130 (2019) 247-265. https://doi.org/10.1016/j.ijimpeng.2019.04.019.

[20] K. Meena, S. Singamneni, A new auxetic structure with significantly reduced stress concentration effects, Mater. Des. 173 (2019) 107779.

https://doi.org/10.1016/j.matdes.2019.107779.

[21] Y. Prawoto, Seeing auxetic materials from the mechanics point of view: A structural review on the negative Poisson's ratio, Comput. Mater. Sci. 58 (2012) 140-153. https://doi.org/10.1016/j.commatsci.2012.02.012.

[22] K.E. Evans, A. Alderson, Auxetic materials: Functional materials and structures from lateral thinking!, Adv. Mater. 12 (2000) 617-628. https://doi.org/10.1002/(SICI)15214095(200005)12:9<617::AID-ADMA617>3.0.CO;2-3.

[23] Y. Jiang, B. Rudra, J. Shim, Y. Li, Limiting strain for auxeticity under large compressive Deformation: Chiral vs. re-entrant cellular solids, Int. J. Solids Struct. 162 (2019) 87-95. https://doi.org/10.1016/j.ijsolstr.2018.11.035.

[24] N. Novak, M. Vesenjak, S. Tanaka, K. Hokamoto, Z. Ren, Compressive behaviour of chiral auxetic cellular structures at different strain rates, Int. J. Impact Eng. 141 (2020) 103566. https://doi.org/10.1016/j.ijimpeng.2020.103566.

[25] N. Novak, L. Krstulović-Opara, Z. Ren, M. Vesenjak, Compression and shear behaviour of graded chiral auxetic structures, Mech. Mater. 148 (2020). https://doi.org/10.1016/j.mechmat.2020.103524.

[26] W. Lee, Y. Jeong, J. Yoo, H. Huh, S.J. Park, S.H. Park, J. Yoon, Effect of auxetic structures on crash behavior of cylindrical tube, Compos. Struct. 208 (2019) 836-846. https://doi.org/10.1016/j.compstruct.2018.10.068.

[27] Y. Guo, J. Zhang, L. Chen, B. Du, H. Liu, L. Chen, W. Li, Y. Liu, Deformation behaviors and energy absorption of auxetic lattice cylindrical structures under axial crushing load, Aerosp. Sci. Technol. 98 (2020) 105662. https://doi.org/10.1016/j.ast.2019.105662.

[28] C. Körner, Y. Liebold-Ribeiro, A systematic approach to identify cellular auxetic materials, Smart Mater. Struct. 24 (2015) 025013. https://doi.org/10.1088/0964-1726/24/2/025013.

[29] V.H. Carneiro, H. Puga, Axisymmetric auxetics, Compos. Struct. 204 (2018) 438-444. https://doi.org/10.1016/j.compstruct.2018.07.116.

[30] W. Zhang, R. Neville, D. Zhang, F. Scarpa, L. Wang, R. Lakes, The two-dimensional elasticity of a chiral hinge lattice metamaterial, Int. J. Solids Struct. 141-142 (2018) 254-263. https://doi.org/10.1016/j.ijsolstr.2018.02.027.

[31] S. Tabacu, R.F. Negrea, D. Negrea, Experimental, numerical and analytical investigation of 2D tetra-anti-chiral structure under compressive loads, Thin-Walled Struct. 155 (2020) 106929. https://doi.org/10.1016/j.tws.2020.106929.

[32] N.D. Tabacu S, Negrea R, Experimental, numerical and analytical investigation of 2D tetraanti-chiral structure under compressive loads, Thin-Walled Struct. 155 (2020). https://doi.org/10.1016/j.tws.2020.106929.

[33] W. Wu, Y. Tao, Y. Xia, J. Chen, H. Lei, L. Sun, D. Fang, Mechanical properties of hierarchical anti-tetrachiral metastructures, Extrem. Mech. Lett. 16 (2017) 18-32. https://doi.org/10.1016/j.eml.2017.08.004.

[34] L.L. Hu, Z.R. Luo, Z.Y. Zhang, M.K. Lian, L.S. Huang, Mechanical property of re-entrant anti-trichiral honeycombs under large deformation, Compos. Part B Eng. 163 (2019) 107-120. https://doi.org/10.1016/j.compositesb.2018.11.010.

[35] X. Yuan, M. Chen, Y. Yao, X. Guo, Y. Huang, Z. Peng, B. Xu, B. Lv, R. Tao, S. Duan, H. Liao, K. Yao, Y. Li, H. Lei, X. Chen, G. Hong, D. Fang, Recent progress in the design and fabrication of multifunctional structures based on metamaterials, Curr. Opin. Solid State Mater. Sci. 25 (2021) 100883. https://doi.org/10.1016/j.cossms.2020.100883.

[36] N. Novak, L. Starčevič, M. Vesenjak, Z. Ren, Blast response study of the sandwich composite panels with 3D chiral auxetic core, Compos. Struct. 210 (2019) 167-178.

https://doi.org/10.1016/j.compstruct.2018.11.050. 